\title{
DESEMPENHO COMUNICATIVO EM TRIGÊMEOS PREMATUROS
}

\section{Acquisition and development language in premature triplets}

\author{
Amanda Tragueta Ferreira (1), Marcela Maria Alves da Silva ${ }^{(2)}$, Luciana Silva ${ }^{(3)}$, \\ Luciana Biral Mendes Merighi (4), Andreza Monforte Miranda ${ }^{(5)}$, \\ Luciana Paula Maximino De-Vitto ${ }^{(6)}$, Dionísia Aparecida Cusin Lamônica ${ }^{(7)}$
}

\section{RESUMO}

Objetivo: descrever habilidades do desenvolvimento de trigêmeos aos 18 meses e aos 29 meses de vida, enfocando a comunicação. Métodos: irmãos trigêmeos dizigóticos do sexo masculino. Os procedimentos de avaliação englobaram: Anamnese, Observação do Comportamento Comunicativo e Escala de Desenvolvimento de Gesell e Amatruda (2000). As avaliações foram realizadas aos 18 e aos 29 meses. As crianças apresentaram atraso do desenvolvimento neuropsicomotor e eram expostas a multilingüismo. Resultados: foi verificada alteração nos comportamentos comunicativos nas três crianças, tanto na primeira quanto na segunda avaliação, embora tenha sido observada melhora do desempenho, após as orientações recebidas pela família. Na segunda avaliação foi observada criptofasia. Dos comportamentos motor grosseiro, delicado, adaptativo, pessoal-social e de linguagem, o último foi o mais afetado para as três crianças, apesar de todos estarem alterados considerando a idade cronológica dos trigêmeos. Conclusão: as habilidades do desenvolvimento dos trigêmeos avaliados neste estudo estavam alteradas, acometendo todas as áreas. Ressalta-se maior comprometimento da linguagem tanto aos 18 como aos 29 meses.

DESCRITORES: Trigêmeos; Linguagem; Prematuro; Desenvolvimento Infantil; Multilinguismo

(1) Fonoaudióloga; Clínica de Fonoaudiologia da Faculdade de Odontologia de Bauru da Universidade de São Paulo.

(2) Fonoaudióloga; Hospital de Reabilitação de Anomalias Craniofaciais da Universidade de São Paulo; Mestranda do Programa de Pós-Graduação Interunidades em Bioengenharia da Universidade de São Paulo, Campus São Carlos.

(3) Fonoaudióloga; Hospital de Reabilitação de Anomalias Craniofaciais da Universidade de São Paulo; Mestre em Fonoaudiologia pela Faculdade de Odontologia de Bauru da Universidade de São Paulo.

(4) Fonoaudióloga; Clínica Long Life; Mestre em Fonoaudiologia pela Faculdade de Odontologia de Bauru da Universidade de São Paulo.

(5) Fonoaudióloga; Escola de Enfermagem de Ribeirão Preto da Universidade de São Paulo; Mestranda em Enfermagem em Saúde Pública pela Escola de Enfermagem de Ribeirão Preto da Universidade de São Paulo.

(6) Fonoaudióloga; Professora Doutora do Departamento de Fonoaudiologia da Faculdade de Odontologia de Bauru da Universidade de São Paulo; Doutora em Ciências Biológicas em Genética Médica e Humana pelo Instituto de Biociências da Universidade Estadual Paulista "Julio de Mesquita Filho" de Botucatu.

(7) Fonoaudióloga; Professora Associada do Departamento de Fonoaudiologia da Faculdade de Odontologia de Bauru da Universidade de São Paulo; Livre-Docente em Distúrbios da Comunicação Humana pela Faculdade de Odontologia de Bauru da Universidade de São Paulo.

\section{INTRODUÇÃO}

Os nascimentos múltiplos têm ocorrido com maior freqüência em todo mundo na última década, devido aos tratamentos de fertilidade ${ }^{1,2}$, trazendo maiores riscos para alterações do desenvolvimento global, incluindo atrasos no desenvolvimento de linguagem ${ }^{3-7 .}$

Há diversas hipóteses referidas pela literatura para atraso de linguagem em casos de nascimentos múltiplos. $\mathrm{O}$ atraso de linguagem pode estar relacionado aos fatores de risco pré, peri e pós-natais $5,6,8$ que podem levar a atraso do desenvolvimento neuropsicomotor. Como a criança aprende a linguagem no intercâmbio com o meio, pela sua exploração ativa e relações que estabelece no seu ambiente, a presença de atraso do desenvolvimento motor é também fator de risco relevante para atraso do desenvolvimento da linguagem ${ }^{9,10}$. A vinda simultânea de mais de uma criança ao lar pode acarretar relativa desvantagem social por aumentar o tamanho da família e trazer dificuldades econômicas. Na verdade, estes fatores, provavelmente, não são inteiramente independentes um do outro ${ }^{6,8}$. 
No que se refere aos aspectos interacionais, estudos têm apresentado duas hipóteses para explicar a alteração de linguagem em nascimentos múltiplos. A primeira afirma que os adultos dispõem de prazos finitos de tempo de atenção para a interação com mais de uma criança em fase de aquisição de linguagem. Assim, o atraso de linguagem em gêmeos ocorreria em função das dificuldades de desdobramento da atenção, da vigilância e dos comentários verbais entre as crianças cuja atenção é ainda pouco seletiva ${ }^{7,8,11,12}$. A segunda aponta que a estreita relação entre os irmãos reduz a necessidade de desenvolvimento verbal e diminui as oportunidades e a motivação para se comunicar 8,13 .

Uma das áreas de interesse relacionada à linguagem gemelar refere-se a um fenômeno conhecido como "linguagem secreta" ou criptofasia ${ }^{8}$. Em nascimentos múltiplos, devido ao contexto social e relação próxima entre os irmãos, pode aparecer a "linguagem secreta", que seria uma forma de interpretação da possível imaturidade de fala das crianças ${ }^{6}$.

No desenvolvimento da linguagem são relatadas duas fases, a fase pré-lingüística que ocorre quando a criança inicia suas vocalizações de fonemas, podendo persistir até por volta de dez a doze meses; e a fase lingüística que se inicia quando a criança fala as primeiras palavras isoladas, com compreensão ${ }^{14-16}$.

$\mathrm{Na}$ fase pré-lingüística a criança inicia o balbucio, imita sons, produz jargão e compreende ordens simples com auxílio de gestos e palavras familiares associadas a ordens simples. A fase lingüística é mais extensa, ocorrendo após os 12 meses de idade. Nesta, aproximadamente com dezoito meses, a criança começa a combinar duas palavras e poderá ter de 30 a 40 elementos em seu vocabulário; conhece algumas partes do corpo; encontra objetos a pedido e apresenta brincadeira simbólica com objetos. Deve apresentar as funções instrumental, regulatória, interacional e pessoal. Aos vinte e quatro meses tem vocabulário de cerca de 150 palavras usando combinações de dois ou três elementos e segue instruções envolvendo dois conceitos verbais. Ocorre mudança na direção da funcionalidade e no avanço na aquisição do vocabulário e da estrutura sintática ${ }^{15-18}$.

Este estudo foi realizado com o intuito de descrever habilidades do desenvolvimento de trigêmeos aos 18 meses e aos 29 meses de vida, enfocando a comunicação.

\section{MÉTODOS}

O estudo descreve o resultado de avaliação fonoaudiológica de três crianças, trigêmeas dizigóticas, do sexo masculino, que chegaram à Clínica de Fonoaudiologia da Faculdade de Odontologia de Bauru - Universidade de São Paulo (FOB/USP), aos 18 meses de idade com queixa de atraso para a fala. As avaliações foram realizadas quando as crianças estavam com 18 meses e elas foram reavaliadas aos 29 meses.

O inventário de anamnese utilizado baseouse no protocolo informativo utilizado na Clínica da FOB/USP, composto das seguintes categorias de informações: dados pessoais, informações gerais sobre a gravidez e nascimento, informações sobre a primeira infância e estágios de desenvolvimento.

A linguagem foi avaliada por meio da Observação do Comportamento Comunicativo (OCC) em atividades lúdicas de cada criança e entre os irmãos e foi utilizada a Escala de Desenvolvimento de Gesell e Amatruda ${ }^{19} \mathrm{em}$ sua totalidade. As categorias de análise desta escala referem-se aos Comportamentos Adaptativo, Motor Grosseiro e Motor Delicado, de Linguagem e Pessoal-Social. A análise desta escala seguiu os procedimentos do manual do avaliador.

Ressalta-se que as sessões da OCC foram filmadas para posterior análise, seguindo a proposta acima apresentada. Cada sessão teve duração média de cinqüenta minutos, totalizando dez sessões para cada criança, englobando avaliação e reavaliação. Todas as crianças foram avaliadas pelos mesmos fonoaudiólogos, os quais também analisaram os dados obtidos.

Após a primeira avaliação, na devolutiva dos resultados, os pais foram orientados verbalmente quanto ao uso de multilingüismo no lar, procedimentos para estimulação da linguagem e aconseIhados a inserirem as crianças na escola, o mesmo ocorrendo após a reavaliação.

Cumpriram-se, na execução deste estudo, os princípios legais conforme versa a Resolução 196/196 do CONEP e a pesquisa foi aprovada pelo Comitê de Ética em Pesquisa, protocolo 113/2007, respeitando todos os aspectos éticos necessários para pesquisas dessa natureza.

Os resultados da avaliação do comportamento e da aplicação da Escala serão apresentados de maneira descritiva e em forma de tabela, respectivamente.

\section{RESULTADOS}

A história clínica revelou fecundação in vitro. Os trigêmeos nasceram de 29 semanas. A primeira criança a nascer pesava 1430 gramas (C1), a segunda 1275 gramas (C2) e a terceira 1550 gramas (C3). O intervalo entre um nascimento e outro foi de, aproximadamente, 2 minutos. Quanto ao nível sócio econômico, os participantes pertencem à classe média superior. 


\begin{tabular}{|cccc|}
\hline Desenvolvimento motor & C1 & C2 & C3 \\
\hline Equilíbrio cervical & $6 \mathrm{~m}$ & $4 \mathrm{~m}$ & $6 \mathrm{~m}$ \\
Engatinhar & - & $7 \mathrm{~m}$ & $8 \mathrm{~m}$ \\
Marcha & $14 \mathrm{~m}$ & $12 \mathrm{~m}$ & $12 \mathrm{~m}$ \\
\hline
\end{tabular}

Legenda (-) ausência. Idade em meses

Figura 1 - Dados do desenvolvimento motor quanto ao equilíbrio cervical, engatinhar e marcha considerando o mês de ocorrência

Os dados relacionados ao desenvolvimento motor dos trigêmeos estão descritos na Figura 1.

Ressalta-se que a família costumava utilizar as línguas portuguesa, inglesa e coreana com os filhos, durante as atividades de vida diária, uma vez que a família apresenta descendência coreana e muitos familiares residem nos Estados Unidos. A maior exposição das crianças com a língua portuguesa era com as três funcionárias cuidadoras.

A mãe relatou que, apesar de saber que gêmeos freqüentemente atrasam para falar, foi orientada a procurar o atendimento, visto que as três crianças apresentaram, conforme relato, atraso no desenvolvimento neuropsicomotor (fizeram tratamento de fisioterapia) e comunicação própria.

Resultados da primeira avaliação - 18 meses:

Durante a avaliação da linguagem, por meio da observação comportamental, verificou-se:

C1: Raros momentos de intenção comunicativa por meio de gestos e apenas função comunicativa instrumental e de protesto. Apresentou raras vocalizações não articuladas. Não chamou a atenção do outro e desistiu das atividades propostas quando surgia algum obstáculo. Não se referiu às situações concretas nem aos objetos presentes. Não atendeu quando chamado pelo nome e compreendeu de modo assistemático às ordens situacionais acompanhadas ou não de gestos. Apresentou dificuldade em imitar gestos e brincadeiras realizadas pelo fonoaudiólogo. Explorou objetos por meio de ações de modo superficial, levando todos à boca (fase sensório-motora). Fez uso convencional de alguns objetos como, por exemplo, bola e carro. Demonstrou agitação e tempo de atenção insuficiente nas atividades programadas. Não fez uso de expressões sociais para iniciar ou encerrar a interação.
C2: Raros momentos de intenção comunicativa por meio de gestos e apenas função comunicativa instrumental (puxa e aponta). Apresentou raras vocalizações não articuladas. Não chamou a atenção do outro e desistiu das atividades propostas quando surgia algum obstáculo. Não se referiu às situações concretas nem a objetos. Não atendeu quando chamado pelo nome, mas compreendeu ordens situacionais acompanhadas de gestos. Apresentou dificuldade em imitar gestos e brincadeiras realizadas pelo fonoaudiólogo. Explorou objetos por meio de poucas ações de modo superficial, levando todos à boca (fase sensório-motora). Não fez uso convencional de objetos. Demonstrou agitação e tempo de atenção insuficiente nas atividades programadas. Não fez uso de expressões sociais para iniciar ou encerrar a interação.

C3: Raros momentos de intenção comunicativa por meio de gestos e apenas função comunicativa instrumental. Apresentou raras vocalizações articuladas. Não chamou a atenção do outro e desistiu das atividades propostas quando surgia algum obstáculo. Não se referiu às situações concretas nem a objetos. Atendeu quando chamado pelo nome e compreendeu ordens situacionais acompanhadas ou não de gestos. Apresentou dificuldade em imitar gestos e brincadeiras realizadas pelo fonoaudiólogo. Explorou objetos por meio de poucas ações de modo superficial, levando todos à boca (fase sensório-motora). Fez uso convencional de alguns objetos como, por exemplo, bola e carro. Demonstrou tempo de atenção insuficiente nas atividades programadas. Fez uso de expressões sociais para iniciar ou encerrar a interação (tchau).

Quanto à observação do comportamento entre os irmãos, verificaram-se comportamentos direcionados um ao outro e verbalizações; não compartiIhavam brinquedos e manipulavam os objetos sem organização. C1 era imitado pelos outros irmãos quando este corria pela sala. C3 solicitava, por meio de gestos, colo do adulto quando não atingia seus objetivos.

Após a análise da aplicação da Escala de Gesell e Amatruda (2000), foram descritos, na Tabela 1, os resultados relacionados às habilidades avaliadas.

Tabela 1 - Resultados da aplicação da Escala de Gesell e Amatruda (2000) das crianças C1, C2 e C3 aos 18 meses $(\mathrm{m})$

\begin{tabular}{c|ccc}
\hline Escala de Gesell \& Amatruda & C1 & C2 & C3 \\
\hline Comportamento motor grosseiro & $15 \mathrm{~m}$ & $18 \mathrm{~m}$ & $15 \mathrm{~m}$ \\
Comportamento motor delicado & $15 \mathrm{~m}$ & $15 \mathrm{~m}$ & $15 \mathrm{~m}$ \\
Comportamento adaptativo & $15 \mathrm{~m}$ & $15 \mathrm{~m}$ & $15 \mathrm{~m}$ \\
Comportamento pessoal-social & $15 \mathrm{~m}$ & $18 \mathrm{~m}$ & $18 \mathrm{~m}$ \\
Comportamento de linguagem & $12 \mathrm{~m}$ & $12 \mathrm{~m}$ & $12 \mathrm{~m}$ \\
\hline
\end{tabular}


Resultados da segunda avaliação - 29 meses:

Durante a avaliação da linguagem, por meio da observação comportamental, verificou-se:

C1: intenção comunicativa por meio de gestos e função comunicativa instrumental e de protesto. Apresentou raras vocalizações articuladas. Chamou a atenção do outro por meio de gestos e desistiu das atividades propostas quando surgia algum obstáculo. Não se referiu às situações concretas nem a objetos. Não atendeu quando chamado pelo nome, compreendeu ordens situacionais simples acompanhadas ou não de gestos. Apresentou menor dificuldade em imitar gestos e brincadeiras realizadas pelo fonoaudiólogo, comparado com a primeira avaliação. Explorou brinquedos de maneira funcional e fez uso convencional de alguns objetos. Demonstrou agitação, mas tempo de atenção suficiente para atividades programadas. Fez uso de expressões sociais para iniciar ou encerrar a interação.

C2: Observou-se intenção comunicativa por meio de gestos e função comunicativa instrumental e de protesto. Apresentou vocalizações articuladas. Chamou a atenção do outro e desistiu das atividades propostas quando surgia algum obstáculo. Não se referiu às situações concretas nem a objetos. Atendeu quando chamado pelo nome e compreendeu ordens situacionais complexas acompanhadas de gestos. Apresentou menor dificuldade em imitar gestos e brincadeiras realizadas pelo fonoaudiólogo, comparado com a primeira avaliação. Explorou brinquedos de maneira funcional e fez uso convencional de alguns objetos. Demonstrou agitação, mas tempo de atenção suficiente para atividades programadas. Fez uso de expressões sociais para iniciar ou encerrar a interação.

C3: Observou-se intenção comunicativa por meio de gestos e função comunicativa instrumental e de protesto. Apresentou vocalizações articuladas. Chamou a atenção do outro e desistiu das atividades propostas quando surgia algum obstáculo. Não se referiu às situações concretas nem a objetos. Atendeu quando chamado pelo nome e compreendeu ordens situacionais complexas acompanhadas ou não de gestos. Apresentou menor dificuldade em imitar gestos e brincadeiras realizadas pelo fonoaudiólogo, comparado com a primeira avaliação. Explorou brinquedos de maneira funcional e fez uso convencional de alguns objetos. Demonstrou tempo de atenção suficiente para atividades programadas. Fez uso de expressões sociais para iniciar ou encerrar a interação.

Quanto à observação do comportamento entre os irmãos, verificaram-se comportamentos direcionados um ao outro, com verbalizações caracterizadas como criptofasia. Compartilhavam brinquedos e manipulavam os objetos com uso funcional.

Após a análise da aplicação da Escala de Gesell e Amatruda (2000), foram descritos, na Tabela 2, os resultados relacionados às habilidades avaliadas.

Após os primeiros contatos com a família, esta foi orientada sobre a importância e a maneira de estimular a comunicação de seus filhos e principalmente quanto à utilização de três idiomas em casa.

Tabela 2 - Resultados da aplicação da Escala de Gesell e Amatruda (2000) nas crianças C1, C2 e C3 aos 29 meses $(\mathrm{m})$

\begin{tabular}{c|ccc}
\hline Escala de Gesell \& Amatruda & C1 & C2 & C3 \\
\hline Comportamento motor grosseiro & $24 \mathrm{~m}$ & $24 \mathrm{~m}$ & $21 \mathrm{~m}$ \\
Comportamento motor delicado & $21 \mathrm{~m}$ & $24 \mathrm{~m}$ & $24 \mathrm{~m}$ \\
Comportamento adaptativo & $21 \mathrm{~m}$ & $24 \mathrm{~m}$ & $24 \mathrm{~m}$ \\
Comportamento pessoal-social & $21 \mathrm{~m}$ & $24 \mathrm{~m}$ & $24 \mathrm{~m}$ \\
Comportamento de linguagem & $15 \mathrm{~m}$ & $18 \mathrm{~m}$ & $21 \mathrm{~m}$ \\
\hline
\end{tabular}

\section{DISCUSSÃO}

O estudo de caso em questão trata-se de trigêmeos que apresentam atraso no desenvolvimento da linguagem. A literatura tem apontado a possibilidade de ocorrência de atraso na aquisição e desenvolvimento de linguagem em crianças advindas de nascimentos múltiplos 2,3,5,6. Como o desenvolvimento da linguagem envolve múltiplos processos, sua evolução está na dependência destas interações nas quais fatores biológicos, sociais e psico- lógicos fazem parte. Neste sentido vale relatar que os irmãos apresentaram outros fatores de risco associados, como prematuridade e baixo peso. Os trigêmeos estudados nasceram de 29 semanas e apresentaram peso entre 1275 a $1550 \mathrm{~g}$. Recémnascido pré-termo, ou prematuro é definido como aquele nascido com idade gestacional abaixo de 37 semanas e criança de baixo peso, como toda aquela nascida viva com peso menor de 2.500 gramas no momento do nascimento ${ }^{4}$. 
Os trigêmeos mesmo tendo iniciado estimulação motora ainda nos primeiros meses de vida, apresentaram atraso motor. Como a linguagem se estabelece no intercâmbio da criança com o seu ambiente, na exploração ativa dos objetos, pelas ações e eventos vivenciados, deve-se considerar a influência do atraso do desenvolvimento neuropsicomotor no desenvolvimento da linguagem. Na presença de atraso a criança terá mais dificuldade de agir com independência nos ambientes nos quais os processos interativos e dialógicos acontecem. De fato, estudos relatam influência do desenvolvimento motor para o desenvolvimento da linguagem ${ }^{9,10}$.

Foi verificada alteração nos comportamentos comunicativos nas três crianças, tanto na primeira quanto na segunda avaliação do comportamento comunicativo, embora tenha sido observada melhora do desempenho após as orientações recebidas pela família. Na segunda avaliação foi observada criptofasia. A criptofasia apesar de ser fenômeno apresentado como corriqueiro na comunicação de gêmeos, não está bem descrita na literatura, entretanto, uma hipótese forte para isto é de que ela não seria uma linguagem verdadeira capaz de ser analisada, mas uma forma de sinais íntimos verbais e não verbais com significados compartilhados pelos irmãos desde o período pré-verbal, época na qual o significado advém do reconhecimento e intercâmbios compartilhados e repetidos ${ }^{6,8}$.

Dos comportamentos motor grosseiro, delicado, adaptativo, pessoal-social e de linguagem (Tabelas 1 e 2), o último foi o mais afetado para as três crianças, apesar de todos estarem atrasados considerando a idade cronológica dos trigêmeos e a expectativa do desenvolvimento de tais habilidades. Conforme a literatura, o ato motor elicia a práxis que favorece a interação da criança com os objetos, eventos e situações nas quais as relações interativas e de aprendizagem acontece ${ }^{9}$, desta forma, o atraso motor traz interferência nas habilidades sociais, pessoais e lingüísticas ${ }^{9,10,19}$.

$\mathrm{Na}$ primeira avaliação da escala de desenvolvimento (Tabela 1), observou-se que os comportamentos motor adaptativo, delicado e linguagem apresentaram escores rebaixados, para as três crianças. Os comportamentos motor grosseiro e pessoal-social também estavam alterados nas crianças C1 e C3. Na segunda avaliação (Tabela 2), foi observada melhora substancial nos comportamentos motor grosseiro, delicado, adaptativo, pessoal-social e linguagem de C2 e C3. Entretanto, abaixo do esperado, considerando a idade cronológica. Cabe enfatizar que a qualidade da estimulação realizada, as oportunidades de interações sociais familiares e extra-familiares, além de fato- res maturacionais provavelmente influenciaram na aquisição das habilidades observadas, conforme também apresenta a literatura ${ }^{1,5-7,10,15}$.

Neste estudo clínico, chama atenção o multilingüismo presente na vida das crianças, com a exposição destas às línguas inglesa, coreana e portuguesa. As influências sociais, econômicas e ambientais no desenvolvimento de habilidades são relatadas na literatura ${ }^{7,17}$. Autores destacaram que quando uma segunda língua é aprendida, outro sistema lexical é desenvolvido, entretanto há um encadeamento destes sistemas conceituais por meio da interpretação de equivalentes do sistema lexical da primeira língua. A criança exposta à língua necessita experienciá-la efetivamente para que consiga armazenar as informações e ir paulatinamente organizando o corpo do conhecimento daquele idioma. A presença de outros fatores de risco para atraso do desenvolvimento juntamente com o multilingüismo pode, entretanto, não favorecer o desenvolvimento pleno da língua mãe, na qual os contextos sociais e culturais estarão envolvidos ${ }^{18}$. No entanto, considerações de que crianças estimuladas em mais de um idioma em idades iniciais do desenvolvimento tendem a responder efetivamente a esta estimulação $e$ conseguir ganhos importantes em habilidades são apresentadas em outros estudos ${ }^{1,11}$.

Uma questão que vale ressaltar é a influência na aquisição de habilidades de linguagem em crianças que freqüentam pré-escolas ou creches. Entretanto, a família, apesar de reconhecer a importância da escola para os filhos, não providenciou o ingresso destes, mas por outro lado, recebeu orientações de como estimular os filhos regularmente. Relataram que promoviam as habilidades de comunicação e brincadeiras entre as crianças, ressaltando que houve mudança na postura dos pais e cuidadoras, inclusive com o uso mais regular do idioma português pelos pais.

Quanto às habilidades lingüísticas, o ganho observado na segunda avaliação refere-se à intenção comunicativa, ou seja, apresentando comportamentos dirigidos a outros, iniciando interações verbais por meio de vocalizações isoladas. Embora este ganho seja evidente, o desempenho observado está aquém do esperado, uma vez que a expectativa nesta fase e que estivessem combinando palavras, conforme atesta a literatura ${ }^{14-16}$. Cabe ressaltar que estudos com gêmeos corroboram tal achado acrescentado de que o desenvolvimento de irmãos provenientes de gestações múltiplas nem sempre é homogêneo, considerando os fatores de risco e maturação individuais ${ }^{12,13}$.

A estreita relação interativa entre as crianças foi observada neste estudo, principalmente a partir da segunda avaliação, não só por meio da constatação 
da linguagem própria entre os trigêmeos, mas também evidenciada pelos relatos familiares. De fato a literatura apresenta a ocorrência de criptofasia em nascimentos múltiplos, relatando esta como uma repercussão desfavorável sobre o processo de individualidade e desempenho comunicativo ${ }^{3,6,8}$. Ressalta-se, entretanto, que há poucos estudos sobre este fenômeno e a influência geral no desenvolvimento da linguagem.

Há muitos fatores de risco para o desenvolvimento pleno em nascimentos múltiplos, mas apesar das evidências para atraso do desenvolvimento, características peculiares como a criptofasia carecem de estudos adicionais para a compreensão deste processo. Crianças nascidas em gestações múltiplas devem ser acompanhadas desde os primórdios de seu desenvolvimento, em caráter preventivo para alterações do desenvolvimento global e da comunicação.

\section{CONCLUSÃO}

$\mathrm{Na}$ descrição das habilidades do desenvolvimento verificou-se atraso nas habilidades avaliadas. Aos 18 meses o atraso do desenvolvimento nas diferentes habilidades é evidente e aos 29 meses ainda observa-se atraso no desenvolvimento da linguagem e criptofasia.

\section{ABSTRACT}

Purpose: to describe abilities of triplets' development by 18 months and the 29 months of life, focusing on communication. Methods: dizygotic male sibling triplets. The evaluation procedures included history of disease, observing the communicative behavior and Escala de Desenvolvimento de Gesell e Amatruda (2000). The evaluations were accomplished by the 18 months and the 29 months. The children showed delay in the neuropshycomotor development and were exposed to multilingualism. Results: alteration was verified in the communicative behaviors in the three children, both in the first as well as in the second evaluation, although an amelioration was shown in the performance, after the orientations received by the family. Cryptophasia was observed in the second evaluation. Of the gross motor, fine motor-adaptive, personal-social and language behaviors, the last one was the most affected as for the three children, in spite of all of them being altered considering the triplets' chronological age. Conclusion: the abilities of the triplets' development appraised in this study were altered, affecting all the areas. Larger commitment of the language is emphasized both by the age of 18 months as well as by 29 months.

KEYWORDS: Triplets; Language; Infant, Premature; Child Development; Multilingualism

\section{REFERÊNCIAS}

1. Feldman R, Eidelman Al. Parent-infant synchrony and the social-emotional development of triplets. Dev Psychol. 2004; 40(6):1133-47.

2. Ricci AP, Perucca E, Pérez CC, Gonzáles VD, Valenzuela RS, Galleguillos FI. Embarazo triple: complicaciones maternas y perinatales. Rev Chil Obst Ginecol. 2005; 70(5):281-8.

3. Bishop DV, Bishop SJ, Bright P, James C, Delaney $T$, Tallal $P$. Different origin of auditory and phonological processing problems in children with language impairment: evidence from a twin study. $J$ Speech Lang Hear Res. 1999; 42(1):155-68.

4. Alegría A, Pittaluga E, Mena P, Schlack L, Dias $\mathrm{MM}$, Vergara SD, et al. Evolución neurosensorial en recién nacido de muy bajo peso de nacimiento a los 2 años de edad corregida. Rev Chil Pediatr. 2002; 37(4):348-56.
5. Hay DA, Preedy P. Meeting the educational needs of multiple birth children. Early Hum Dev. 2006; 82(6):397-403.

6 . Thorpe K. Twin children's language development. Early Hum Dev. 2006; 82(6):387-95.

7. Sutcliffe AG, Derom C. Follow-up of twins: health, behaviour, speech, language outcomes and implications for parents. Early Hum Dev. 2006; 82(6):379-86.

8. Mogford K. Desenvolvimento de linguagem em gêmeos. In: Bishop D, Mogfors $\mathrm{K}$, organizador. Desenvolvimento da linguagem em circunstâncias exepcionais. Rio de Janeiro: Revinter; 2002. p. 99-122.

9. Lamônica DAC. Linguagem na paralisia cerebral. In: Ferreira LP, Befi-Lopes DM, Limongi SCO. Tratado de fonoaudiologia. São Paulo: Roca; 2004. p. 967-76. 
10. Webster RI, Majnemer A, Platt RW, Shevell MI. Motor function at school age in children with a preschool diagnosis of developmental language impairment. J Pediatr. 2005; 146(1):80-5.

11. Alves TE, Franco KEVB, Hage SRV. Habilidades conversacionais de crianças gêmeas: influência da encefalopatia bilirrubínica. Rev CEFAC. 2004; 6(3):253-8.

12. Stromswold K. Why aren't identical twins linguistically identical? Genetic, prenatal and postnatal factors. Cognition. 2006; 101(2):333-84.

13. Rutter M, Thorpe K, Greenwood R, Northstone $\mathrm{K}$, Golding J. Twins as a natural experiment to study the causes of mild language delay: I: Design; twinsingleton differences in language, and obstetric risks. J Child Psychol Psychiatry. 2003; 44(3):326-41.

14. Tomasello M, Kruger AC. Joint attention on actions: acquiring verbs in ostensive and non-ostensive contexts. J Child Lang. 1992; 19(2):311-33.
15. Costa DI, Azambuja LA, Nunes ML. Avaliação do desenvolvimento neuropsicomotor. In: Nunes $\mathrm{ML}$, Marroni ACH. Semiologia neurológica. Porto Alegre: EDIPUCRS; 2002. p. 351-60.

16. Schirmer CR, Fontoura DR, Nunes ML. Distúrbios da aquisição da linguagem e da aprendizagem. J Pediatr. 2003; 80(2):95-103.

17. Kendal L. Social, economic and environmental influences on disorders of hearing, language and speech. J Commun Disord. 2005; 38(1):261-2.

18. Devitto Z, Burguess C. Theoretical and methodological implications of language experience and vocabulary skill: priming of strongly and weakly associated words. Brain Cogn. 2004; 55(2):295-9.

19. Gesell A, Amatruda CS. Diagnóstico do desenvolvimento: avaliação do desenvolvimento neuropsicológico no latente e na criança pequena: o normal e o patológico. Rio de Janeiro: Atheneu; 2000.

RECEBIDO EM: 12/09/2007

ACEITO EM: 04/02/2008

Endereço para correspondência:

Rua Caetano Sampieri, 7-60, ap. 24

Bauru - SP

CEP: $17011-420$.

Tel: (14)3226-3092 / (14)9721-0848 / (18)9705-4711

E-mail: atf_fono_usp@yahoo.com.br 